\section{Qualitative assessment of undetectable melanin distribution in lightly pigmented irides}

\author{
Gladimir V. G. Baranoski and Michael W. Y. Lam \\ University of Waterloo, School of Computer Science, \\ Natural Phenomena Simulation Group, 200 University \\ Avenue West, Waterloo, Ontario, Canada N2L 3G1
}

\begin{abstract}
A longstanding issue in biomedical research concerns the biophysical factors affecting the color diversity of the human irides and its connection with ocular diseases. Although the pigmentation and morphological characteristics of heavily and moderately pigmented irides have been extensively discussed in the literature, similar studies are scarce for lightly pigmented irides. We present computer experiments indicating that the spectral signature of these specimens may be directly affected by still undetectable melanin distributions in the outermost iridal layers. Our findings represent in silico evidence of this relationship which, in turn, may have implications in the investigation of the higher risk of death from metastatic ocular melanoma verified in individuals with light-colored irides. () 2007 Society of Photo-Optical Instrumentation Engineers. [DOI: $10.1117 / 1.2747205]$
\end{abstract}

Keywords: iris; reflectance; melanin; melanoma; tissue optics.

Paper 06344LR received Nov. 28, 2006; revised manuscript received Feb. 12, 2007; accepted for publication Feb. 28, 2007; published online Jun. 15, 2007.

The iris can be schematically described as a multilayered tissue stretching across the front of the eye and surrounding the pupil. The outermost (or frontmost) iridal layer is the anterior border layer $(\mathrm{ABL})$. It consists of a dense arrangement of pigmented cells, collagen fibers, and fibroblasts. ${ }^{1}$ Immediately behind the ABL, we find the stromal layer (SL). Although both layers consist of connective tissue and pigmented cells, the SL is less dense than the ABL. ${ }^{1}$ The SL is also characterized by the presence of loosely arranged collagen fibrils. The inner most layer is an opaque tissue called the iris pigment epithelium (IPE). It consists of heavily pigmented epithelial cells that are tightly fused by intercellular connections. $^{2}$

Several studies ${ }^{1-3}$ indicate that the pigmentation of the innermost iridal layer, the IPE, does not vary significantly between irides of different colors. Such spectral differences are mainly determined by the presence (or absence) of light absorbing pigments, notably the brown-black melanin (eumelanin) and red-yellow melanin (pheomelanin), in the two outermost tissues, namely the ABL and the SL. Wet measurements involving these tissues ${ }^{4}$ indicate that brown irides have a high content of both pigments, whereas blue irides exhibit a low content of either pigment. We remark, however, that other

Address all correspondence to Gladimir Baranoski, Computer Science, University of Waterloo, 200 University Avenue West-Waterloo, Ontario N2L sG1 Canada, Tel: 1-519-8884567; E-mail: gvgbaran@curumin.math.uwaterloo.ca natural pigments, such as hemoglobins ${ }^{5}$ (oxygenated and deoxygenated) and carotenoids ${ }^{6}$ (lutein and zeaxanthin) found in the SL, are also known to contribute to the spectral signature of the human iris. Furthermore, light traversing the iridal tissues can be scattered by stromal collagen fibrils in a Rayleigh fashion ${ }^{7}$ producing blue hues in lightly pigmented irides.

Spectral data obtained using reflectometry indicate that the light interactions with iridal tissues result in discrete families of spectral signatures, and it was suggested that such interactions may be affected by the distribution and size of melanin particles within these tissues. ${ }^{5}$ In terms of the effects of melanin distribution on the iridal spectral signatures, it is assumed that the appearance of iridal colors other than blue is directly related to the melanin content in the ABL. ${ }^{3,8}$ Recent measurements performed on blue irides using electron spin resonance spectroscopy detected less melanin in medium blue irides than in light blue irides. ${ }^{9}$ It was postulated that this surprising finding could be related to the localization of pigment granules in the outermost iridal layers. ${ }^{9}$ Difficulties ${ }^{4,9}$ in performing separate wet measurements on the ABL and SL have precluded the in loco verification of this hypothesis, however.

Computational simulations are being employed in a variety of fields to assist the study of complex biological systems, ${ }^{10-12}$ and several computer models ${ }^{13-17}$ have been proposed to investigate the processes that control the amount of light that enters the human eye and its interaction with ocular fundus tissues. Recently, we developed the first biophysically based iridal light transport model (ILIT) to investigate tissue optics phenomena affecting iridal appearance. The detailed description of this model, including the evaluation of its quantitative predictions through comparisons of modeled results with actual measured data, is provided elsewhere. ${ }^{18}$

Briefly, the ILIT model uses ray optics and Monte Carlo methods to simulate light transport within the human iris. Although each ray is associated with a wavelength, it is assumed that the energies of different wavelengths are decoupled. The light interactions with the iridal tissues are modeled as a stochastic process whose states are represented by the interface between the iris and the surrounding medium, as well as the interfaces between adjacent iridal tissues (ABL, SL, and IPE). A ray traversing from one iridal layer to the next must pass the interface between these layers. At these interfaces, the rays can be reflected or transmitted. The results of these interactions are associated with the transition probabilities of the stochastic process: they are used to select the next layer that the ray will traverse, thus its next state. Scattering events affecting the direction of propagation of a given ray may also trigger the transition from one state to another. These events are probabilistically simulated using Rayleigh-based and cosine-based distribution functions. The latter accounts for the diffuse perturbation of the ray due to the internal arrangement of the tissues. Absorption events, which are determined by the presence of pigments in the iridal tissues, provide the termination probabilities to the stochastic process (i.e., when a ray is absorbed its transport simulation is terminated). These events are simulated considering the probability of absorption of a photon (ray) traveling a certain distance at a certain wavelength in the medium ${ }^{10}$ and taking into account the spec-

1083-3668/2007/12(3)/030501/3/\$25.00 @ 2007 SPIE 


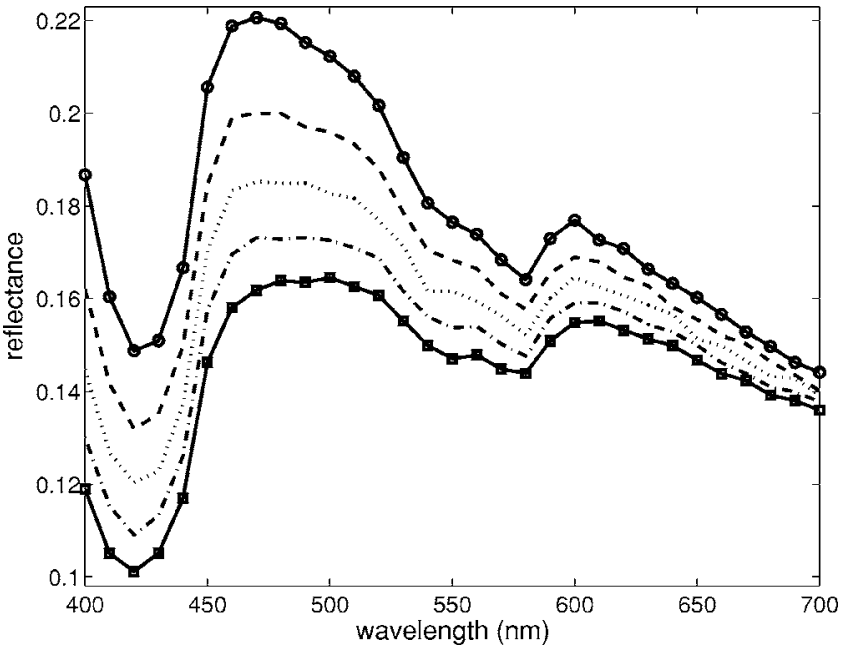

Fig. 1 Changes in the spectral signature of a simulated lightly pigmented iris in response to different PMSL values: 10\% (squares), $30 \%$ (dashed-dotted line), 50\% (dotted line), 70\% (dashed line), and 90\% (circles).

tral absorption coefficients and concentrations of the pigments found within the iridal layers.

Previous ILIT simulations ${ }^{18}$ confirmed the assumption that brown irides have a darker appearance when most of the melanin present in the two outermost iridal layers, ABL and SL, are located in the former. The results of our ILIT simulations involving lightly pigmented irides, however, indicate a reverse relationship between the appearance of these specimens and their melanin distribution. In these simulations, we employed the same iridal data set used in the previous experiments on brown irides (which is also fully disclosed elsewhere ${ }^{18}$ ) with the exception of lower melanin amounts. More specifically, for the simulated lightly pigmented specimen used in these experiments, we considered $3.1 \mu \mathrm{g}$ of eumelanin and $0.5 \mu \mathrm{g}$ of pheomelanin present in the ABL and SL, which correspond to values derived from measurements described in the literature. ${ }^{4}$

The key biophysical parameter in these simulations is the percentage of melanin in the SL, henceforth referred to as PMSL. It corresponds to the ratio between the amount of melanin in the SL and the total amount of melanin in both ABL and SL. As we increase the PMSL, several changes are observed in the spectral signature of the simulated lightly pigmented iris. The most noticeable is the reflectance increase across the visible spectral domain (Fig. 1), with reflectance values obtained at shorter wavelengths showing increase rates steeper than those associated to values obtained at longer wavelengths (Fig. 2). Another change refers to the characteristic $\mathrm{W}$ hemoglobin signature, ${ }^{5}$ determined by the absorption bands of oxyhemoglobin at 542 and $577 \mathrm{~nm},{ }^{19}$ which becomes skewed within this spectral interval (Fig. 1). Finally, the reflectance peak tends to be shifted toward shorter wavelengths (Fig. 1). These changes suggest that the lighter shades and the hue transitions toward blue observed in lightly pigmented irides may be associated to a relatively higher content of melanin in the SL, which may be also related to existence of light blue irides with melanin contents higher than those measured for medium blue irides. ${ }^{9}$ To illustrate the latter pos-

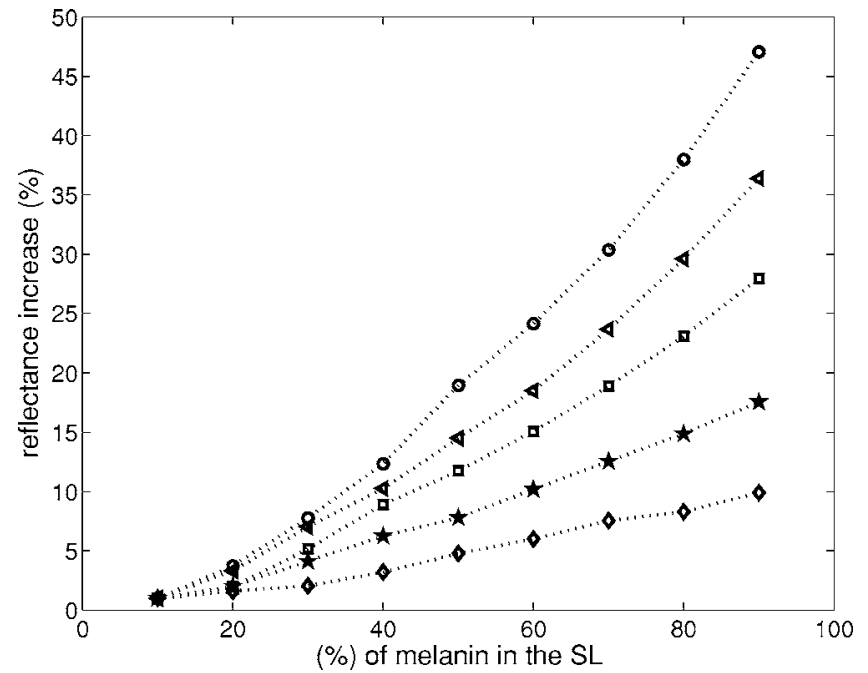

Fig. 2 Reflectance increase rates of a simulated lightly pigmented iris with respect to PMSL variations. The circles, triangles, squares, stars, and diamonds represent modeled data obtained at 420, 470, 510, 560 , and $630 \mathrm{~nm}$, respectively.

sibility, we performed another experiment in which we considered two simulated lightly pigmented specimens A and B. For specimen A, we kept the same melanin amounts and set the PMSL to $70 \%$. For specimen B, we reduced the melanin amounts in the ABL and SL by $10 \%$, and we set the PMSL to $50 \%$. The resulting spectral curves (Fig. 3) show a lower reflectance profile for specimen B despite the higher melanin content of specimen A.

Epidemiological and statistical studies indicate that individuals with light-colored irides have a higher risk of developing ocular melanoma ${ }^{20,21}$ and a lower probability of surviving the more aggressive metastatic form of this disease. ${ }^{22,23}$ Nevertheless, the interdependence between iris color and the metastatic spread, via blood vessels, of ocular melanomas remains unexplained. It is worth noting, however, that the color

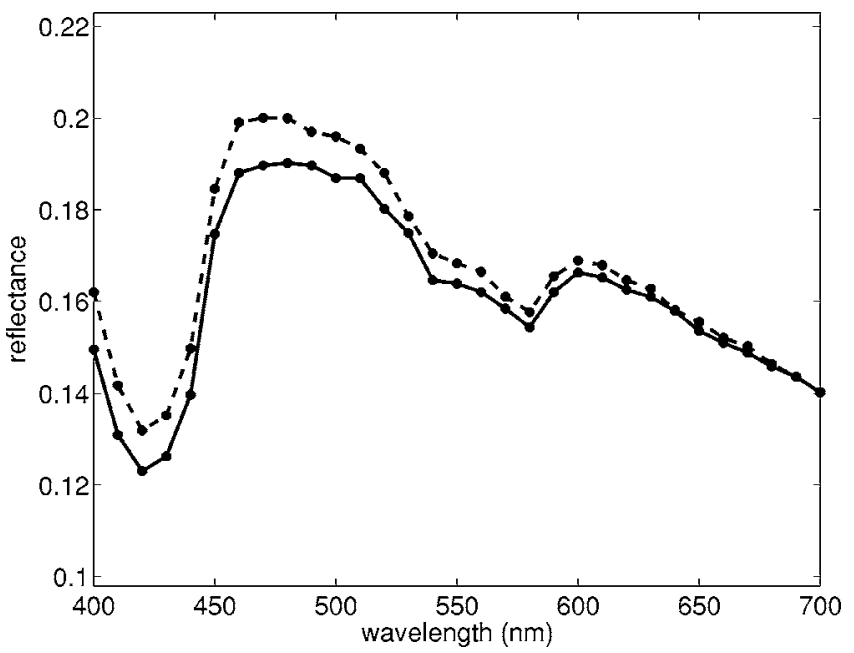

Fig. 3 Comparison of reflectance curves for two simulated lightly pigmented specimens A and B. For specimen A (dashed line), the PMSL was set to $70 \%$. For specimen B (solid line), the PMSL was set to $50 \%$, and the $\mathrm{ABL}$ and $\mathrm{SL}$ melanin amounts were reduced by $10 \%$. 


\section{JBO LETTERS}

classification methods used in these studies do not incorporate measurements indicating melanin content and distribution. ${ }^{5,9}$ The results of our simulations suggest that not only the small amount of melanin within the outermost iridal layers, but also its possible predominant presence in the blood irrigated SL should be taken into account in future investigations of biophysical processes responsible for iridal melanoma. In addition, our findings highlight the potentiality of using computational experiments in conjunction with reflectometry tools and wet measurement technologies to trace photobiological factors $^{24}$ responsible for the pathogenesis of ocular melanomas.

\section{Acknowledgments}

The authors would like to thank the anonymous reviewers for their helpful comments. This work was supported by the Natural Sciences and Engineering Research Council of Canada (NSERC Grant No. 238337) and the Canada Foundation for Innovation (CFI Grant No. 33418).

\section{References}

1. R. C. Eagle, "Iris pigmentation and pigmented lesions: An ultrastructural study," Trans. Am. Ophthalmol. Soc. 88, 581-687 (1988).

2. P. D. Imesh, I. H. L. Wallow, and D. M. Albert, "The color of the human eye: A review of morphological correlates and some conditions that affect iridal pigmentation," Surv. Ophthalmol. 41(2), 117123 (1997)

3. P. D. Imesh, C. D. Bindley, Z. Khademian, B. Ladd, R. Gangmon, D. M. Albert, and I. H. L. Wallow, "Melanocytes and iris color," Arch. Ophthalmol. (Chicago) 114, 443-447 (1986).

4. G. Prota, D. Hu, M. R. Vincensi, S. A. McCormick, and A. Napolitano, "Characterization of melanins in human irides and cultured uveal melanocytes from eyes of different colors," Exp. Eye Res. 67, 293-299 (1998)

5. F. C. Delori, C. K. Dorey, and K. A. Fitch, "Characterization of ocular melanin by iris reflectometry," Invest. Ophthalmol. Visual Sci. 32(1-4), 1144 (1991).

6. P. S. Bernstein, F. Khachik, L. S. Carvalho, G. J. Muir, D. Zhao, and N. B. Katz, "Identification and quantification of carotenoids and their metabolites in the tissues of the human eye," Exp. Eye Res. 72, 215223 (2001).

7. C. L. Wilkerson, N. A. Syed, M. R. Fischer, N. L. Robinson, I. H. L. Wallow, and D. M. Albert, "Melanocytes and iris colors," Arch. Ophthalmol. (Chicago) 114, 437-442 (1996).
8. I. A. Menon, D. C. Wakeham, S. D. Persad, M. Avaria, G. E. Trope, and P. K. Basu, "Quantitative determination of the melanin contents in ocular tissues from human blue and brown eyes," J. Ocul. Pharmacol. 8(1), 35-42 (1992).

9. A. R. Wielgus and T. Sarna, "Melanin in human irides of different color and age of donors," Pigment Cell Res. 18, 454-464 (2005).

10. S. A. Prahl, M. Keijzer, S. L. Jacques, and A. J. Welch, "A Monte Carlo model of light propagation in tissue," SPIE Inst. Ser. IS 5, 102-111 (1989).

11. V. Tuchin, Tissue Optics Light Scattering Methods and Instruments for Medical Diagnosis, SPIE Press, Bellingham (2000).

12. B. D. Ventura, C. Lemerle, K. Michalodimitrakis, and L. Serrano, "From in vivo to in silico biology and back," Nature (London) $\mathbf{4 4 3}$ 527-533 (2006).

13. D. van Norren and L. F. Tiemeijer, "Spectral reflectance of the human eye," Vision Res. 26(2), 313-320 (1986).

14. F. C. Delori and K. P. Pflibsen, "Spectral reflectance of the human ocular fundus," Appl. Opt. 28, 1061-1077 (1989).

15. M. Hammer, A. Roggant, D. Schweitzer, and G. Muller, "Optical properties of ocular fundus tissues-and in vitro study using the double-integrating sphere technique and inverse Monte Carlo simulation," Phys. Med. Biol. 40, 963-978 (1995).

16. M. Hammer and D. Schweitzer, "Quantitative reflection spectroscopy at the human ocular fundus," Phys. Med. Biol. 47, 179-191 (2002).

17. S. J. Preece and E. Claridge, "Monte Carlo modelling of the spectral reflectance of the human eye," Phys. Med. Biol. 47, 2863-2877 (2002).

18. M. W. Y. Lam and G. V. G. Baranoski, "A predictive light transport model for the human iris," Comput. Graph. Forum 25(3), 359-368 (2006).

19. G. Zonios, J. Bykowski, and N. Kollias, "Skin melanin, hemoglobin, and light scattering properties can be quantitatively assessed in vivo using diffuse reflectance spectroscopy," J. Invest. Dermatol. 117, 1452-1457 (2001)

20. A. Stang, W. Ahrens, G. Anastassiou, and K. Jöckel, "Phenotypical characteristics, lifestyle, social class and uveal melanoma," Ophthalmic Epidemiol. 105, 293-302 (2003).

21. A. D. Singh, I. G. Rennie, S. Seregard, M. Giblin, and J. McKenzie, "Sunlight exposure and pathogenesis of uveal melanoma," Surv. Ophthalmol. 49(4), 419-428 (2004).

22. S. Regan, K. M. Egan, and E. S. Gragoudas, "Iris color as a risk factor in uveal melanoma," Invest. Ophthalmol. Visual Sci. 38(4), 810 (1997).

23. S. Regan, H. E. Judge, E. S. Gragoudas, and K. M. Egan, "Iris color as a prognostic factor in ocular melanoma," Arch. Ophthalmol. (Chicago) 117, 811-814 (1999).

24. D. Hu, "Photobiology of ocular melanocytes and melanoma," Photochem. Photobiol. 81, 506-509 (2005). 